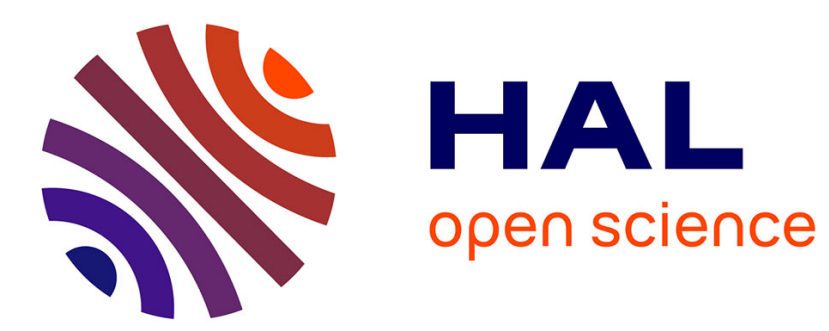

\title{
Performances of Weighted Cyclic Prefix OFDM with Low-Complexity Equalization
}

\author{
Damien Roque, Cyrille Siclet
}

\section{To cite this version:}

Damien Roque, Cyrille Siclet. Performances of Weighted Cyclic Prefix OFDM with Low-Complexity Equalization. IEEE Communications Letters, 2013, IEEE Communications Letters, 17 (3), pp.439442. 10.1109/LCOMM.2013.011513.121997 . hal-01260517

\section{HAL Id: hal-01260517 https://hal.science/hal-01260517}

Submitted on 25 Jan 2016

HAL is a multi-disciplinary open access archive for the deposit and dissemination of scientific research documents, whether they are published or not. The documents may come from teaching and research institutions in France or abroad, or from public or private research centers.
L'archive ouverte pluridisciplinaire HAL, est destinée au dépôt et à la diffusion de documents scientifiques de niveau recherche, publiés ou non, émanant des établissements d'enseignement et de recherche français ou étrangers, des laboratoires publics ou privés. 


\title{
Performances of Weighted Cyclic Prefix OFDM with Low-Complexity Equalization
}

\author{
Damien Roque and Cyrille Siclet
}

\begin{abstract}
In this paper, we justify low-complexity equalization techniques for weighted cyclic prefix (WCP)-OFDM. This modulation technique refers to filter bank based multicarrier (FBMC) transmission system provided with short filters. It allows the use of non-rectangular waveforms in order to mitigate interference caused by time-frequency selective channels while preserving an efficient implementation.
\end{abstract}

Index Terms-time-varying multipath channels, filter bank based multicarrier modulations, equalization, efficient realization.

\section{INTRODUCTION}

Mobile radio applications in terrestrial environment usually imply multipath propagation and motion-induced Doppler spread. Such a channel may be modeled as a time-frequency spreading operator. Under narrowband assumptions, it simplifies to a linear time-varying (LTV) system. The approximate eigenstructure of LTV systems has been discussed in [1]. This study reveals the need of pulse shaped multicarrier systems in order to match the time-frequency characteristics of doubly selective channels.

A practical implementation of the resulting transceiver relies on filter bank based multicarrier (FBMC) systems. Efficient realization schemes have been proposed in [2], providing orthogonal sub-channels and making use of fast Fourier transform (FFT) algorithm. Apart from the implementation, the design of pulse-shaping filters is an active area of research [3]-[5].

Since the pulse shapes are usually longer than each data block, the underlying transceiver requires a polyphase decomposition and advanced equalization techniques. Consequently, despite attractive performances results [6], FBMC systems are not widely used because of their relative complexity compared to traditional block transmission frameworks.

Therefore, the most common multicarrier scheme remains cyclic prefix orthogonal frequency-division multiplexing (CPOFDM) scheme. It is based on a rectangular pulse-shaping and presents the advantage of diagonalizing time-invariant channels if a guard interval longer than the channel impulse response is used. Assuming invertibility of this eigensystem, perfect reconstruction of the transmitted symbols is performed using a single-tap per sub-channel equalizer [7]. However, the Doppler spread introduced by time-variant channels breaks orthogonality between sub-channels, resulting in inter-carrier interference (ICI).

D. Roque is with the Direction Générale de l'Armement and GIPSA-lab, e-mail: damien.roque@gipsa-lab.grenoble-inp.fr.

C. Siclet is with the GIPSA-lab, Grenoble.
In this work, we focus on short pulse shape filters, whose impulse response is shorter than data blocks [8]. This particular class of FBMC system is referred to as weighted cyclic prefix orthogonal frequency-division multiplexing (WCP-OFDM). Such a generalization of CP-OFDM through the use of non-rectangular filters offers an interesting trade-off between channel-induced interference mitigation and complexity [9].

Previous work has shown that WCP-OFDM outperforms CP-OFDM for several mobile radio environment with a singletap per sub-channel equalizer [10]. We propose here morethan-one coefficient per sub-channel equalization scenarios, keeping a linear inversion complexity and show that WCPOFDM still outperforms CP-OFDM using these scenarios.

\section{GENERAL FRAMEWORK}

In this section, we describe the input-output relation of a WCP-OFDM transmultiplexer (fig. 1) in presence of a doubly selective channel.

\section{A. WCP-OFDM transmultiplexer structure}

Let $\boldsymbol{c}_{n}=\left[c_{0, n} \ldots c_{M-1, n}\right]^{T}$ be the $n$th block of $M$ complex symbols, with ${ }^{T}$ being the transpose operator. The corresponding block at the output of the transmitter consists in $N$ samples $\boldsymbol{s}_{n}=\left[s_{n}[0] \ldots s_{n}[N-1]\right]^{T}$, with $\Delta=N-M \geq 1$. Given $\gamma[k]=0$ if $k<0$ or $k>N-1$, the transmitted signal fullfils the relation

$$
s_{n}[k]=\frac{1}{\sqrt{M}} \sum_{m=0}^{M-1} c_{m, n} \gamma[k] e^{j \frac{2 \pi m k}{M}} .
$$

If we let $\boldsymbol{F}_{M}$ be the $M$-size discrete Fourier transform (DFT) matrix with entries $F_{M}[k, l]=1 / \sqrt{M} \exp (-j 2 \pi k l / M)$ for $0 \leq k, l \leq M-1$ and $\boldsymbol{P}_{\Delta}$ the $N \times M$ cyclic extension matrix with entries $P_{\Delta}[k, m]=\delta_{k, m}+\delta_{k-M, m}$ for $0 \leq k \leq N-1$ and $0 \leq m \leq M-1$ we may write in a similar way

$$
\boldsymbol{s}_{n}=\boldsymbol{D}_{\gamma} \boldsymbol{P}_{\Delta} \boldsymbol{F}_{M}^{H} \boldsymbol{c}_{n}
$$

where ${ }^{H}$ denotes the transpose conjugate operation and $\boldsymbol{D}_{\gamma}=$ $\operatorname{diag}(\gamma[0], \ldots, \gamma[N-1])$.

Let $\boldsymbol{r}_{n}=\left[r_{n}[0] \ldots r_{n}[N-1]\right]^{T}$ be the $n$th block of $N$ received symbols. The corresponding $M$ estimated symbols are given by

$$
\tilde{c}_{m, n}=\frac{1}{\sqrt{M}} \sum_{k=0}^{N-1} r[k] \check{\gamma}[k] e^{-j \frac{2 \pi m k}{M}} .
$$




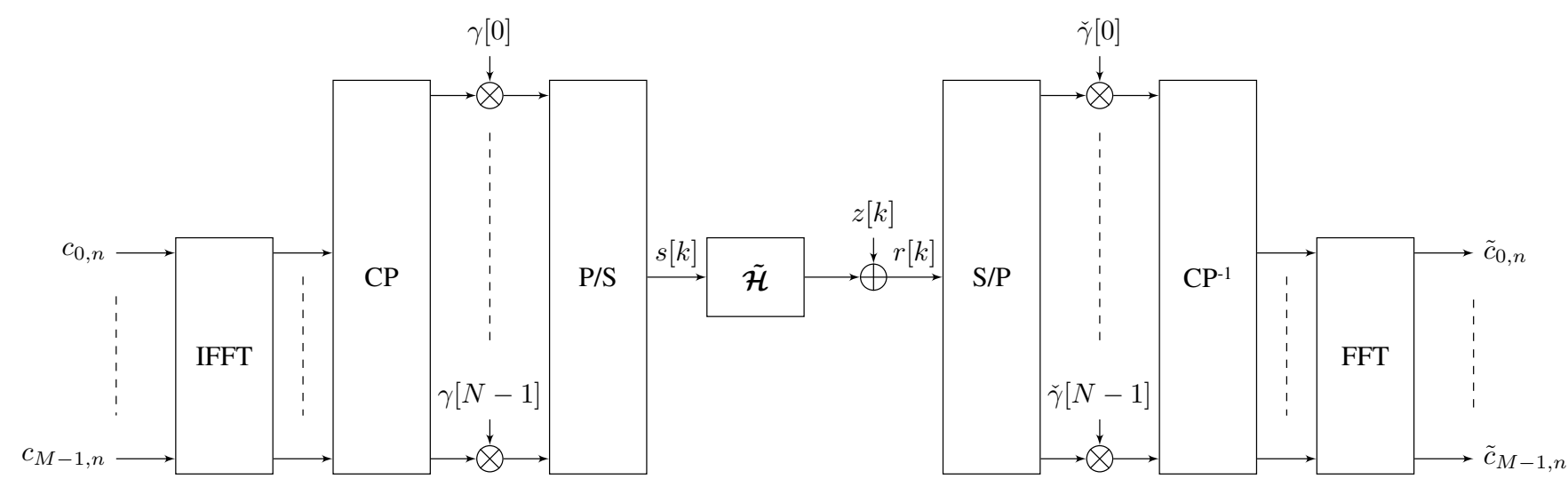

Fig. 1: Efficient implementation of a WCP-OFDM transmultiplexer.

If we denote $\boldsymbol{D}_{\gamma}=\operatorname{diag}(\gamma[0], \ldots, \gamma[N-1])$, we can write equivalently

$$
\tilde{\boldsymbol{c}}_{n}=\boldsymbol{F}_{M} \boldsymbol{P}_{\Delta}^{T} \boldsymbol{D}_{\check{\gamma}} \boldsymbol{r}_{n} .
$$

In the case of a perfect channel without noise, namely if $\boldsymbol{r}_{n}=\boldsymbol{s}_{n}$, the combination of (2) and (4) gives

$$
\tilde{\boldsymbol{c}}_{n}=\boldsymbol{F}_{M} \boldsymbol{P}_{\Delta}^{T} \boldsymbol{D}_{\check{\gamma}} \boldsymbol{D}_{\gamma} \boldsymbol{P}_{\Delta} \boldsymbol{F}_{M}^{H} \boldsymbol{c}_{n} .
$$

Since $\boldsymbol{F}_{M}^{H}=\boldsymbol{F}_{M}^{-1}$, the perfect reconstruction (PR) of the transmitted symbols is achieved when

$$
\boldsymbol{P}_{\Delta}^{T} \boldsymbol{D}_{\check{\gamma}} \boldsymbol{D}_{\gamma} \boldsymbol{P}_{\Delta}=\boldsymbol{I}_{M}
$$

with $\boldsymbol{I}_{M}$ the $M$-size identity matrix. A PR system is said biorthogonal if $\gamma[k] \neq \check{\gamma}[k]$ and orthogonal if $\gamma[k]=\check{\gamma}[k]$.

As an example, CP-OFDM is a biorthogonal system. The transmitter pulse shape is given by $\gamma^{\mathrm{CP}}[k]=1$ for $0 \leq k \leq$ $N-1$ whereas the receiver pulse shape is written as $\check{\gamma}^{\mathrm{CP}}[k]=0$ for $0 \leq k \leq \Delta-1$ and $\check{\gamma}^{\mathrm{CP}}[k]=1$ for $\Delta \leq k \leq N-1$.

Two orthogonal systems have been proposed in [8], assuming $N / \Delta=M / \Delta+1$ and odd values of $M / \Delta$. One of them is optimized with respect to time-frequency localization (TFL) criterion and the underlying pulse shapes are denoted $\gamma^{\mathrm{TFL}}[k]=\check{\gamma}^{\mathrm{TFL}}[k]$ (fig. 2). Compared to rectangular pulses used in CP-OFDM, a previous study confirms the interest of the TFL criterion for mobile channels [10]. The orthogonal setup also offers an optimal approach over additive white Gaussian noise channels [11, p. 160].
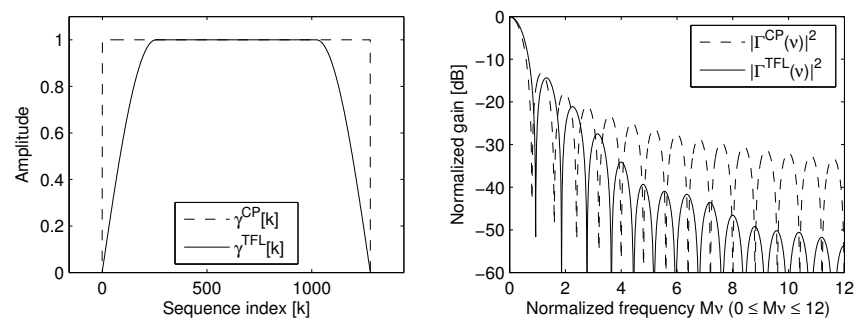

Fig. 2: Time and frequency responses of the prototype filters with $\Gamma(\nu)=1 /\|\gamma\|_{\infty} \sum_{k=0}^{N-1} \gamma[k] \exp (-j 2 \pi \nu k / N)$ and $\|\gamma\|_{\infty}=\max \{|\gamma[k]|\}_{k \in \mathbf{Z}}$, using $M=1024$ and $N=1280$.

\section{B. Multipath time-variant channel model}

In a mobile radio environment, we consider a time-variant channel $\mathcal{H}$, with $I$ resolvable paths. Let $s(t)$ be the baseband equivalent transmitted signal, limited to a band $B$. In a narrowband context, the received signal is given by

$$
r(t)=(\mathcal{H} s)(t)+z(t)=\sum_{i=1}^{I} \alpha_{i}(t) s\left(t-\tau_{i}\right)+z(t)
$$

where $\alpha_{i}(t)$ is a complex gain associated to the $i$ th path, at delay $\tau_{i}$. The signal $z(t)$ is a complex bandlimited Gaussian noise characterised by its power spectral density $2 N_{0}=\sigma_{z}^{2} / B$ for $|f| \leq B / 2$ and 0 otherwise.

In order to relate the channel model to a filter bank transceiver, we express a discrete-time version of $\mathcal{H}$, denoted $\tilde{\mathcal{H}}$. Using the bandlimited hypothesis and if we let $s_{n}[k]=s[k+n N]=s((k+n N) / B), z_{n}[k]=z[k+n N]=$ $z((k+n N) / B)$ and $r_{n}[k]=r[k+n N]=r((k+n N) / B)$, for $0 \leq k \leq N-1$, the input-output relation becomes

$$
r[q]=(\tilde{\mathcal{H}} s)[q]+z[q]=\sum_{l \in \mathbf{Z}} \tilde{\alpha}_{l}[q] s[q-l]+z[q]
$$

with

$$
\tilde{\alpha}_{l}[q]=\sum_{i=1}^{I} \alpha_{i}\left(\frac{q}{B}\right) \operatorname{sinc}\left(B \tau_{i}-l\right) .
$$

Bandlimiting operation leads to an infinite number of coefficients $\tilde{\alpha}_{l}(t)(l \in \mathbf{Z})$. In practice, the sequence is truncated to $L$ taps whenever $\left\{\left|\tilde{\alpha}_{l}(t)\right|^{2}\right\}_{l>L} \approx 0$.

In the context of short filters used by WCP-OFDM, the input-output relation given in (8) may be simplified if $L \leq N$. In other words, if the channel delay is less than the block length, interblock interference (IBI) is restricted to two consecutive blocks and we have

$$
\boldsymbol{r}_{n}=\boldsymbol{H}_{n} \boldsymbol{s}_{n}+\boldsymbol{G}_{n} \boldsymbol{s}_{n-1}+\boldsymbol{z}_{n}, \quad n \in \mathbf{N}
$$

where $\boldsymbol{z}_{n}=\left[z_{n}[0] \ldots z_{n}[N-1]\right]^{T}$. The transfer function of the discrete-time equivalent channel over the $n$th block is 
described by two $N \times N$ matrices defined as

$$
\begin{aligned}
& \boldsymbol{H}_{n}= \\
& {\left[\begin{array}{ccccc}
\tilde{\alpha}_{1}[n N] & 0 & 0 & \cdots & 0 \\
\vdots & \tilde{\alpha}_{1}[n N+1] & 0 & \cdots & 0 \\
\tilde{\alpha}_{L}[n N] & \cdots & \ddots & \cdots & 0 \\
\vdots & \ddots & \cdots & \ddots & 0 \\
0 & \cdots & \tilde{\alpha}_{L}[n N+2] \cdots \tilde{\alpha}_{1}[n N+N-1]
\end{array}\right]}
\end{aligned}
$$

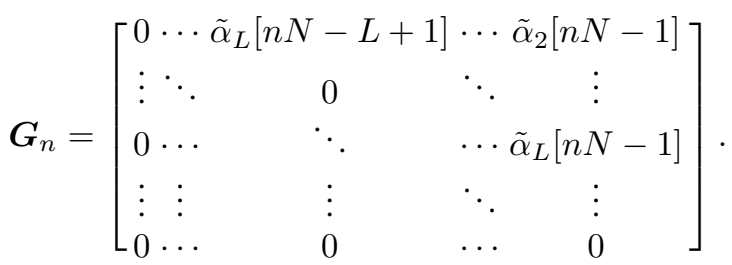

Combining (2), (4) and (10), the overall transmultiplexer inputoutput relation writes

$$
\begin{aligned}
& \tilde{\boldsymbol{c}}_{n}=\underbrace{\boldsymbol{F}_{M} \boldsymbol{P}_{\Delta}^{T} \boldsymbol{D}_{\check{\gamma}} \boldsymbol{H}_{n} \boldsymbol{D}_{\gamma} \boldsymbol{P}_{\Delta} \boldsymbol{F}_{M}^{H}}_{\boldsymbol{A}_{n}} \boldsymbol{c}_{n} \\
& +\underbrace{\boldsymbol{F}_{M} \boldsymbol{P}_{\Delta}^{T} \boldsymbol{D}_{\check{\gamma}} \boldsymbol{G}_{n} \boldsymbol{D}_{\gamma} \boldsymbol{P}_{\Delta} \boldsymbol{F}_{M}^{H}}_{\boldsymbol{B}_{n}} \boldsymbol{c}_{n-1}+\underbrace{\boldsymbol{F}_{M} \boldsymbol{P}_{\Delta}^{T} \boldsymbol{D}_{\check{\gamma}} \boldsymbol{z}_{n}}_{\boldsymbol{\zeta}_{n}} .
\end{aligned}
$$

where $\boldsymbol{\zeta}_{n}$ corresponds to the noise term projection on the receiver and $\boldsymbol{A}_{n}$ and $\boldsymbol{B}_{n}$ represent the WCP-OFDM transfer matrices associated to the $n$th block.

\section{WCP-OFDM EQUALIZATION SCHEMES FOR A GIVEN SIMULATION FRAMEWORK}

As stated above, IBI and ICI may occur in the general case of WCP-OFDM transmultiplexer in presence of a timefrequency selective channel. However, IBI involves at most two consecutive blocks. As a consequence, the analysis of $\mathrm{E}\left\{|A[m, p]|^{2}\right\}$ and $\mathrm{E}\left\{|B[m, p]|^{2}\right\}$ is sufficient to determine the most appropriate equalization scheme for a given application.

In this simulation framework, we consider a QPSK modulated transmission system, using a band $B=8 \mathrm{MHz}$, centered around a frequency $f_{c}=5 \mathrm{GHz}$. We use a 6-path WSSUS channel model where the last path occurs at $5 \mu$ s (COST 207 TUx6 [12]). It implies a highly frequency selective behavior over the band $B$. Two mobility scenarios are developed with regard to the fast fading assumption: pedestrian $\left(v_{\max }=3\right.$ $\mathrm{km} / \mathrm{h})$ and vehicular $\left(v_{\max }=350 \mathrm{~km} / \mathrm{h}\right)$.

Using TFL optimized pulses, figures 3 and 4 confirm that IBI and ICI decrease as $N / M$ increases. As expected, ICI increases with the Doppler spread. Furthermore, IBI is negligible compared to ICI in both mobility scenarios. As a consequence, a single-tap per sub-carrier equalizer may be sufficient in the low mobility scenario whereas ICI from adjacent sub-channels should be mitigated in the high mobility case.

In the following, we consider two low-complexity equalizers and we denote $\overline{\boldsymbol{c}}_{n}$ the equalized signal. Channel impulse response estimation is beyond the scope of this paper.

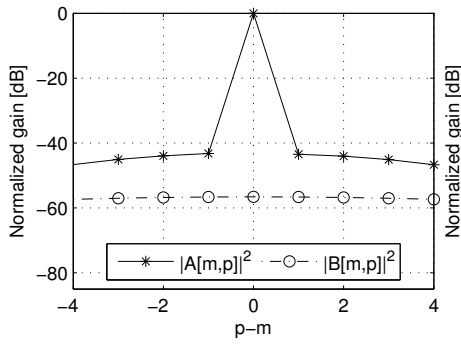

(a) $N / M=9 / 8$.

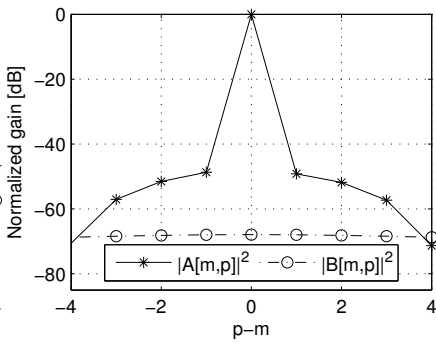

(b) $N / M=5 / 4$.
Fig. 3: WCP-OFDM system mean gains $\mathrm{E}\left\{|A[m, p]|^{2}\right\}$ and $\mathrm{E}\left\{|B[m, p]|^{2}\right\}$ with $M=512, v_{\max }=3 \mathrm{~km} / \mathrm{h}$.

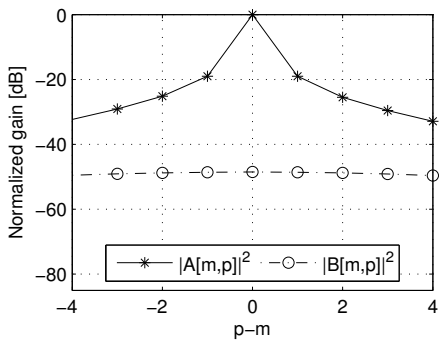

(a) $N / M=9 / 8$.

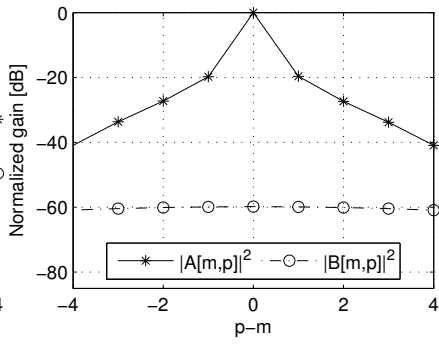

(b) $N / M=5 / 4$.
Fig. 4: WCP-OFDM system mean gains $\mathrm{E}\left\{|A[m, p]|^{2}\right\}$ and $\mathrm{E}\left\{|B[m, p]|^{2}\right\}$ with $M=512, v_{\max }=350 \mathrm{~km} / \mathrm{h}$.

1) Equalizer 1: We first propose a single tap per subchannel equalizer. This approach considers that IBI and ICI terms are negligible. It relies on the approximation of $\boldsymbol{A}_{n}$ as a diagonal matrix written

$$
\tilde{A}_{n}[m, p]=A_{n}[m, p] \delta_{m, p}
$$

Assuming that $\tilde{A}_{n}$ is invertible, the equalized symbols are given by

$$
\overline{\boldsymbol{c}}_{n}=\tilde{\boldsymbol{A}}_{n}^{-1} \tilde{\boldsymbol{c}}_{n}=\tilde{\boldsymbol{A}}_{n}^{-1} \boldsymbol{A}_{n} \boldsymbol{c}_{n}+\tilde{\boldsymbol{A}}_{n}^{-1} \boldsymbol{B}_{n} \boldsymbol{c}_{n-1}+\tilde{\boldsymbol{A}}_{n}^{-1} \boldsymbol{\zeta}_{n} .
$$

This equalizer may lead to perfect reconstruction in the case of a CP-OFDM facing a frequency selective channel, provided a prefix greater than the last echo is used. However, if $\tilde{A}_{n}$ has close-to-zero diagonal terms, noise and interference terms may be amplified. In the general case of WCP-OFDM with a severe time-frequency selective channel, IBI and ICI terms may remain preponderant over the noise term [10].

2) Equalizer 2: In the case of mainly time-selective channels, the major performances degradation is caused by ICI and it becomes interesting to cancel interferences induced by adjacent sub-channels. To this end, we approximate $\boldsymbol{A}_{n}$ as a tridiagonal matrix defined by

$$
\tilde{A}_{n}[m, p]=A_{n}[m, p]\left(\delta_{m, p}+\delta_{m, p-1}+\delta_{m, p+1}\right) .
$$

If $\tilde{A}_{n}$ is invertible, we retrieve the expression given in (15). Thanks to Thomas algorithm [13], the resolution of a tridiagonal system results in a $\mathcal{O}(N)$ operation wich is much more affordable than a general $N \times N$ matrix inversion whose complexity is usually $\mathcal{O}\left(N^{3}\right)$. 


\section{BER SIMULATION RESULTS}

We compare the performances of CP-OFDM and WCPOFDM with TFL pulses using $M=512$ and $N / M=5 / 4$. We focus our analysis on the two equalization scenarios described above. Bit error rate (BER) is plotted as a function of $E_{b} / N_{0}$, where $E_{b}=\sigma_{c}^{2}\|\gamma\|^{2} / 2 M B$ and $N_{0}=\sigma_{z}^{2} / 2 B$ (fig. 5).

When the Doppler spread tends to zero (fig. 5a), ICI is negligible and equalizers 1 and 2 yield the same results. WCP-OFDM with TFL pulses shows better results than CPOFDM in presence of noise, thanks to prototypes functions orthogonality.

In a high mobility scenario (fig. 5b), an interference floor appears at high $E_{b} / N_{0}$ values. It demonstrates the interest of three taps per sub-carrier equalization for both WCPOFDM and CP-OFDM. The clear advantage of WCP-OFDM is justified by the good frequency containment of the TFL pulses.

\section{CONClusion}

The focus of our work on short filters leads to an efficient FBMC modulator-demodulator referred to as WCP-OFDM and whose complexity is similar to traditional cyclic prefix OFDM.

Through the analysis of the transfer matrices of a realistic system, we proposed two block equalization scenarios, preserving a linear inversion complexity.

Finally, BER simulation results confirm the interest of WCP-OFDM with TFL filters with regard to CP-OFDM. This system, provided with one to three coefficients per subchannel, is particularly resilient to ICI in the case of highly time-selective channels.

\section{ACKNOWLEDGMENT}

This work has been supported by the French defense procurement agency (DGA) and Orange-Labs.

\section{REFERENCES}

[1] W. Kozek and A. F. Molisch, "On the eigenstructure of underspread WSSUS channels," in Proc. First IEEE Signal Processing Workshop Signal Processing Advances in Wireless Communications, 1997, pp. 325-328.

[2] C. Siclet, P. Siohan, and D. Pinchon, "Analysis and design of OFDM/QAM and BFDM/QAM oversampled orthogonal and biorthogonal multicarrier modulations," in IEEE ICASSP'02, Orlando, USA, May 2002, student forum.

[3] T. Strohmer and S. Beaver, "Optimal OFDM design for time-frequency dispersive channels," IEEE Trans. Commun., vol. 51, no. 7, pp. 11111122, 2003.

[4] C. Siclet, P. Siohan, and D. Pinchon, "Perfect reconstruction conditions and design of oversampled DFT-modulated transmultiplexers," EURASIP journal on applied signal processing, vol. 2006, pp. 1-14, 2006.

[5] P. Jung and G. Wunder, "WSSUS pulse design problem in multicarrier transmission," IEEE Trans. Commun., vol. 55, no. 9, p. 1822, 2007.

[6] A. M. Tonello and F. Pecile, "Analytical results about the robustness of FMT modulation with several prototype pulses in time-frequency selective fading channels," IEEE Trans. Wireless Commun., vol. 7, no. 5, pp. 1634-1645, 2008.

[7] Z. Wang and G. B. Giannakis, "Wireless multicarrier communications," IEEE Signal Process. Mag., vol. 17, no. 3, pp. 29-48, 2000.

[8] D. Pinchon and P. Siohan, "Closed-form expressions of optimal short PR FMT prototype filters," in Proc. IEEE Global Telecommunications Conf. GLOBECOM'11, 2011.

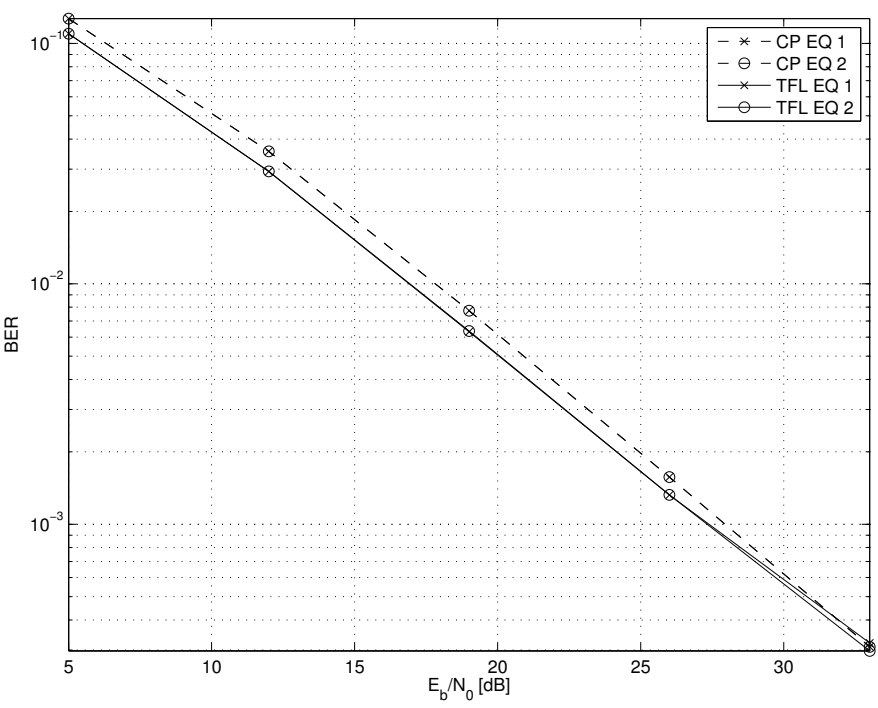

(a) BER performances for $v_{\max }=3 \mathrm{~km} / \mathrm{h}$.

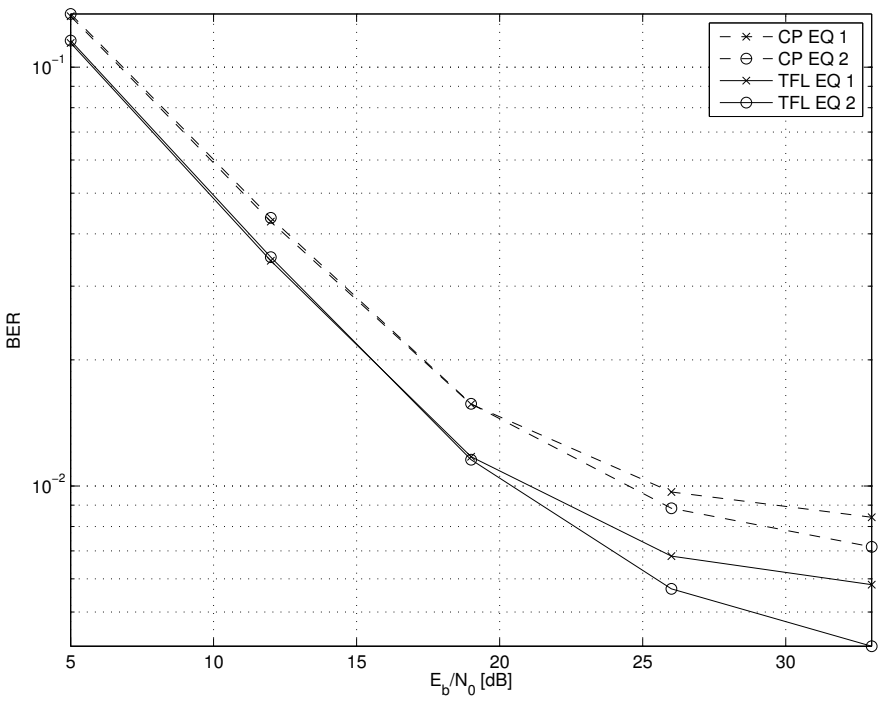

(b) BER performances for $v_{\max }=350 \mathrm{~km} / \mathrm{h}$.

Fig. 5: BER performances for pedestrian and vehicular scenarios in COST 207 TUx6 channel. Comparison between CPOFDM and WCP-OFDM (with TFL pulses) for $M=512$ and $N / M=5 / 4$.

[9] N. Moret and A. M. Tonello, "Design of orthogonal filtered multitone modulation systems and comparison among efficient realizations," EURASIP J. Adv. Signal Process, vol. 2010, pp. 10:1-10:11, January 2010.

[10] D. Roque, C. Siclet, and P. Siohan, "A performance comparison of FBMC modulation schemes with short perfect reconstruction filters," in Proc. IEEE International Conference on Telecommunications, ICT '12, 2012.

[11] J. Proakis and M. Salehi, Digital communications, 5th ed. McGrawHill, 2006.

[12] M. Failli, "COST 207 digital land mobile radio communications," Commission of the European Communities, p. 137, 1988.

[13] S. Conte and C. Boor, Elementary numerical analysis: an algorithmic approach. McGraw-Hill Higher Education, 1980. 